EPiC Series in Engineering
Volume 3, 2018, Pages 1531-1538
HIC 2018. 13th International
Conference on Hydroinformatics

\title{
Scale-invariance generalized logistic (GLO) model for estimating extreme design rainfalls in the context of climate change
}

\author{
Truong-Huy Nguyen ${ }^{1, *}$ and Van-Thanh-Van Nguyen ${ }^{1}$ \\ ${ }^{1}$ Department of Civil Engineering and Applied Mechanics, McGill University, Montreal, Canada. \\ huy.nguyen5@mail.mcgill.ca,van.tv.nguyen@mcgill.ca
}

\begin{abstract}
Statistical models based on the scale-invariance (or scaling) concept has increasingly become an essential tool for modeling extreme rainfall processes over a wide range of time scales. In particular, in the context of climate change these scaling models can be used to describe the linkages between the distributions of sub-daily extreme rainfalls (ERs) and the distribution of daily ERs that is commonly provided by global or regional climate simulations. Furthermore, the Generalized Logistic distribution (GLO) has been recommended in UK for modeling of extreme hydrologic variables. Therefore, the main objective of the present study is to propose a scaling GLO model for modeling ER processes over different time scales. The feasibility and accuracy of this model were assessed using ER data from a network of 21 raingages located in Ontario, Canada. Results of this assessment based on different statistical criteria have indicated the comparable performance of the proposed scaling GLO model as compared to other popular models in practice. Furthermore, an illustrative application of the proposed model for evaluating the climate change impacts on the ERs in Ontario using the available NASA downscaled regional climate simulations has demonstrated the accuracy and robustness of the GLO model.
\end{abstract}

\section{Introduction}

The estimation of extreme design rainfalls in the context of potential climate change impacts has become essential in current engineering practices due to recent recognition of climate variability [14]. This estimation requires hence a suitable rainfall (spatial) downscaling approach to establishing an accurate linkage between daily climate projections from global (or regional) climate models and daily extreme rainfall (ER) processes at a local site [1,5-7]. Furthermore, this linkage so far has been

\footnotetext{
${ }^{*}$ Corresponding author
} 
commonly established at the daily timestep since current climate models have some major limitations in their detailed physical modeling ability and their limited computational capability. Consequently, it requires an improved (temporal) rainfall modeling approach to describe the linkages between the ER processes over a wide range of time scales (e.g., from one day to several minutes) [8-11].

The spatial resolution problems of GCM and RCM outputs has been examined in detail in several previous studies. In particular, several different downscaling methods have been proposed to spatially disaggregate GCM or RCM projected outputs to much finer regional scales or local/point scales (single-site or multi-site cases) for reliable assessments of climate change impacts [5-7, 12, 13-15]. However, very few studies are dealing with the temporal downscaling problem of GCM or RCM outputs. More specifically, some procedures have been proposed to derive the key statistics of the sub-daily ER series from those of the daily series $[9,11]$. Among these methods, the statistical models based on the scale-invariance (or scaling) concept has recently increasingly become an essential tool for modeling ER processes over a wide range of temporal scales [16-19].

Scale invariance implies that the statistical properties of ER over different time scales are related to each other by an operator involving only the scale ratio and the scaling exponent [20]. Applications of the scale-invariance (scaling) concept has begun since the last decades. However, only a few scaleinvariance models have been reported in the literature, including the scaling generalized extreme value (GEV) distribution and its special case, the scaling Gumbel (GUM) distribution [21-24]. These scaling models have been extensively applied to the estimation of short-duration ERs at gauged and ungauged sites, as well as to the construction of IDF curves in the current climate and in a changing climate $[8,22,24-29]$. In practice, nonetheless, there are many different probability models that have been used for describing the distribution of annual rainfall extremes at a single site [30-32], but there is still no general agreement as to which distribution(s) should be used.

In view of the above-mentioned issues, the main objective of the present study is therefore to propose a novel scale-invariance model for modelling accurately the ER process over a wide range of time scales (e.g.,from several minutes to one day). In particular, the generalized logistic model (GLO) has been selected for this study since this distribution has been recommended for frequency analyses of extreme hydrologic variables in the UK $[31,33]$. The scale-invariant property and the mathematical framework for estimating the parameters of the scaling GLO model based on both the non-central moments (NCMs) and the probability weighted moments (PWMs) methods are derived and presented in Section 2. The feasibility and accuracy of the proposed model using these two estimation methods (GLO/PWM and GLO/NCM) are assessed and compared with two popular models (GUM and GEV) using IDF data from a network of 21 raingages located in Ontario, Canada, as described in Sections 3 and 4. A summary of research findings is provided in Section 5.

\section{The scale-invariance GLO model}

\subsection{The GLO distribution}

The GLO distribution has been used for representing the probability distribution of extreme rainfalls and for constructing the rainfall intensity-duration-frequency (IDF) relations. Its cumulative distribution function, $\mathrm{F}(\mathrm{x})$, and the quantile function, $\mathrm{x}(\mathrm{F})$, are defined by [30]:

$$
\begin{aligned}
& F(x)=1 /(1+\exp (-y)) \quad \text { where } \quad y=\left\{\begin{array}{c}
-k^{-1} \log \left\{1-\frac{k(x-\xi)}{\alpha}\right\}, k \neq 0 \\
(x-\xi) / \alpha
\end{array}, k=0\right. \\
& x(F)= \begin{cases}\xi+\alpha \frac{\{(1-F) / F\}^{k}}{k}, & k \neq 0 \\
\xi-\alpha \log \{(1-F) / F\}, & k=0\end{cases}
\end{aligned}
$$

in which $\xi, \alpha$, and $\kappa$ are the location, scale, and shape parameters, respectively. 


\subsection{The scale-invariance GLO/PWM model}

The probability weighted moments (PWMs) and its linear combination forms (L-moments) can be used for estimation of the GLO parameters in consideration of the scaling property of these PWMs with rainfall durations. The $r^{\text {th }}$ order of PWMs, $B_{r}$, of the GLO distribution can be expressed as:

$B_{r}=(r+1)^{-1}\left(\xi+\frac{\alpha}{\kappa}\left\{1-\frac{\Gamma(1+\kappa) \Gamma(r+1-\kappa)}{\Gamma(r+1)}\right\}\right)$

in which $\Gamma($.$) is the gamma function. Therefore, it is possible to estimate parameters (\xi, \alpha, \kappa)$ of the GLO model using the first three PWMs.

For a simple scaling process, it can be shown that: $B_{r}(\lambda t)=\lambda^{\beta_{r}} B_{r}(t)=\lambda^{\beta} B_{r}(t)$

where $\beta_{r}=\beta$, and $\beta=\beta_{0}^{P W M}$ is the scaling exponent or PWM order $r=0$ (i.e. the mean).

\subsection{The scale-invariance GLO/NCM model}

The non-central moment (NCM) method can be used for estimation of the GLO parameters in consideration of the scaling property of these NCMs with rainfall durations. The $r^{\text {th }}$ order of NCMs, $\mu_{r}$, of the GLO distribution can be expressed as:

$\mu_{r}=\left(\xi+\frac{\alpha}{\kappa}\right)^{r}+(-1)^{r}\left(\frac{\alpha}{\kappa}\right)^{r} \Gamma(1+r \kappa) \Gamma(1-r \kappa)+r \sum_{i=1}^{r-1}(-1)^{i}\left(\frac{\alpha}{\kappa}\right)^{i}\left(\xi+\frac{\alpha}{\kappa}\right)^{k-1} \Gamma(1+i \kappa) \Gamma(1-i \kappa)$

in which $\Gamma($.$) is the gamma function. Therefore, it is possible to estimate parameters (\xi, \alpha, \kappa)$ of the GLO model using the first three NCMs.

For a simple scaling process, it can be shown that: $\mu_{r}(\lambda t)=\lambda^{\beta} \mu_{r}(t)=\lambda^{r \beta} \mu_{r}(t)$

where $\beta_{r}=r \beta$, and $\beta=\beta_{1}^{N C M}$ is the scaling exponent or the NCM order $r=1$ (i.e. the mean).

In summary, for a simple scaling process, notice that the statistical properties of either the GLO/PWM or GLO/NCM model for two different time scales $t$ and $\lambda t$ are related as follows:

$\kappa(\lambda t)=\kappa(t) ; \alpha(\lambda t)=\lambda^{\beta} \alpha(t) ; \xi(\lambda t)=\lambda^{\beta} \xi(t) ; X_{T}(\lambda t)=\lambda^{\beta} X_{T}(t) ;$

\section{Numerical application}

\subsection{Study sites and data}

IDF data of nine durations ( 5 minutes to 1440 minutes) from a network of 21 stations located in the Ontario province of Canada were selected for this study. The record lengths for these stations vary from 40 years to 75 years. Selection of these stations relied on the quality of the data, the adequate length of available historical extreme rainfall records, and the spatial distribution of raingages representing diverse climatic conditions of Ontario.

\subsection{Goodness-of-fit assessment criteria}

Six common goodness-of-fit criteria were selected for assessing the feasibility and accuracy of the proposed scale-invariance GLO/PWM and GLO/NCM models and for comparing the performance of these models with those given by existing models. These criteria include the root mean square error (RMSE), the root mean square relative error (RMSEr), the mean absolute deviation (MAD), the mean absolute relative deviation (MADr), the maximum absolute error (MAE), and the correlation coefficient (CC). After computing the six statistical tests, a ranking scheme is utilized to rank all the 
selected distributions. Ranking scores is assigned to each model according to the value computed for each criterion. A distribution with the lowest RMSE, RMSEr, MAD, MADr, MAE, or highest CC is given a rank of 1 for this assessment category. In case of ties, average ranks are given to those corresponding models. Furthermore, for each numerical criterion, the overall rank associated with each distribution is computed by summing the individual rank.

$$
\begin{aligned}
& R M S E=\left\{\sum \frac{\left(x_{i}-y_{i}\right)^{2}}{(n-m)}\right\}^{1 / 2} \quad \text { (8) } \quad R M S E r=\left[\frac{1}{(n-m)} \sum\left\{\frac{\left(x_{i}-y_{i}\right)}{x_{i}}\right\}^{2}\right]^{1 / 2} \\
& M A D=\sum \frac{\left|x_{i}-y_{i}\right|}{(n-m)} \\
& M A E=\max \left(\left|x_{i}-y_{i}\right|\right) \\
& \begin{array}{l}
M A D r=\frac{1}{(n-m)} \sum\left\{\frac{\left|x_{i}-y_{i}\right|}{x_{i}}\right\} \\
C C=\frac{\sum\left\{\left(x_{i}-\bar{x}\right)\left(y_{i}-\bar{y}\right)\right\}}{\left\{\sum\left(x_{i}-\bar{x}\right)^{2} \sum\left(y_{i}-\bar{y}\right)^{2}\right\}^{1 / 2}}
\end{array}
\end{aligned}
$$

where $x_{i}, i=1,2, \ldots, n$ are the observed values and $y_{i}, i=1,2, \ldots, n$ are the values estimated from a scale-invariance model for the same probability level $p_{i} ; m$ is the number of distribution parameters; $\bar{x}$ and $\bar{y}$ denote the average values of the observations and estimated quantiles respectively.

\section{Results and discussions}

For purposes of illustration, only representative results for Ottawa CDA RCS and Toronto International Airport stations are presented here as shown in Figure 1. Both the graphical displays and numerical criteria were used to evaluate the performance of the proposed GLO/PWM and GLO/NCM scale-invariance models. For the graphical displays, the quantile-quantile (Q-Q) plots of the 5-min to 720-min observed and estimated annual maximum series (AMS) are used for the visual comparison. The estimated values are derived from the 1440-min (or 1-day) AMS data using the five scaleinvariance models: GLO/PWM, GLO/NCM, GUM/PWM, GEV/NCM, and GUM/NCM. Results show that all scaling models closely described the left-tail and central parts. The right-tail parts, however, are less well described and there are no obvious trends. These values can be well estimated, over-estimated, or under-estimated by any of the five scaling models. From the graphical comparison basis, all the scale-invariance models seem to perform well for these two stations. However, the significance of the differences between these models is difficult to assess based on the graphical display. A more objective evaluation using numerical comparison criteria is thus necessary.
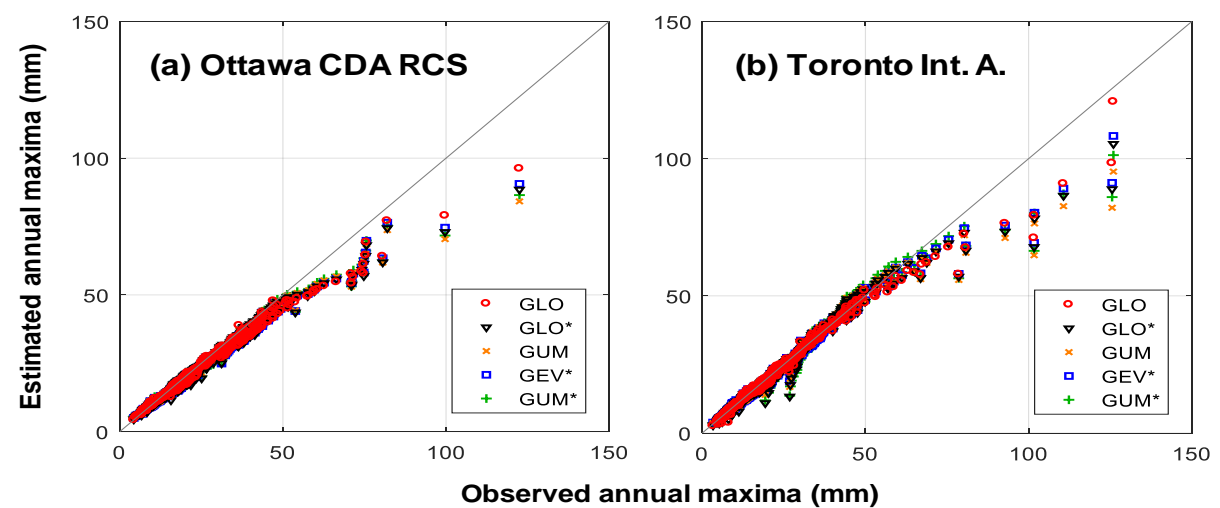

Figure 1: Q-Q plots of the 5-min to 720-min observed and estimated AMS data for (a) Ottawa CDA RCS and (b) Toronto International Airport stations. Note that GLO and GUM denote scaling models based on PWMs while GLO*, GEV* and GUM* denote scaling models based on NCMs. 
Figure 2 shows the results of the six numerical comparison indices for 5-min observed and estimated AMS data. Numerical values of the six indices are displayed in the form of standard boxplots. Note that the GEV and GUM denote the scale-invariance models based on PWMs (i.e., GEV/PWM and GUM/PWM), while the remaining GEV* and GUM* denote the scale-invariance models based on NCMs (i.e., GEV/NCM and GUM/NCM). Results based on the scaled 5-min AMS show that, in general, all five scale-invariance performed well with RMSEr and MADr are less than $15 \%$, RMSE and MAD are less than $1.5 \mathrm{~mm}$, and CC is higher than 0.9. The ranking of the five candidate scaling models for each of the selected 21 stations based on the six indices are also presented. Ranking from number 1 to 5 indicates the gradual decrease from the best to the worst distributions. On the basis of these goodness-of-fit numerical comparison results, it was found that no unique scaling model ranked consistently best for all locations and for all rainfall durations.

Figure 3 shows the overall rank of each scaling model computed for all stations for each rainfall duration and for each performance criterion. Note that the GLO and GUM denote the scale-invariance models based on PWMs (i.e., GLO/PWM and GUM/PWM), while the remaining GLO*, GEV*, and $\mathrm{GUM}^{*}$ denote the scale-invariance models based on NCMs (i.e., GLO/NCM, GEV/NCM, and GUM/NCM). In general, based on most selected criteria, the GLO/NCM is the least accurate while the GEV/NCM is the best model among the five models considered. In addition, the GUM/PWM could perform better than the GEV/NCM for the majority of durations according to the MAD and MADr indices. Furthermore, the proposed GLO/PWM could provide similar results as compared to the GEV/NCM for different durations and criteria, especially for 5-min and 360-min durations. The GLO/PWM also provides comparable results with the GUM/PWM in RMSE, RMSEr criteria for many durations and it performs better than the GUM/PWM in the CC and MAE indices. Therefore, while the GEV/NCM is the best model for all criteria, the comparable performance of the GLO/PWM has indicated that it could be used along with the GEV/NCM to increase the confidence and reliability in estimating extreme design rainfalls in the context of climate change, especially in the UK where the GLO has been recommended.

To test the feasibility and accuracy of the proposed model in constructing future IDF relations, observed data and downscaled global climate model (GCM) outputs from seven selected stations in Ontario were utilized. These climate simulation outputs were produced from 21 (GCMs) and have been downscaled by NASA to a regional $25-\mathrm{km}$ scale [34]. The bias correction approach based on transfer functions (i.e., scaling factors) were applied for transferring the NASA extreme rainfalls at the regional 25-km scale, $\hat{X}$, to a given local site, $X_{i}$ [35]. The observed data from 1961-1990 and 1991-2005 at each site were used for the calibration and validation of the model respectively. Figure 4a shows the probability plots of the computed design extreme rainfalls $X_{\mathrm{T}}(\mathrm{mm})$ for three different durations (i.e. 1-day, 1-hour, and 5-min) at the Winsor station for both the calibration and validation periods. Uncertainty associated with the estimation of the extreme design rainfalls is displayed in the form of boxplots. Results show that the distributions of the estimated sub-daily ERs derived based on the distribution of daily ERs and the scale-invariance GLO/PWM model reflect well the observed data. Figure $4 \mathrm{~b}$ shows the $\mathrm{Q}-\mathrm{Q}$ plots of the estimated extreme design rainfalls derived based on the scale-invariance GLO/PWM and the at-site frequency analysis using the GLO distribution for different rainfall durations and return periods at all seven stations. Note that the median values of the results from $21 \mathrm{GCMs}$ were used for the computation. A numerical comparison using three dimensionless GOF indices (i.e. RMSEr, MADr, and CC) for all seven sites as shown in Table 1. The low values of RMSEr and MADr as well as the high values of CC indicate that the proposed scaling GLO/PWM are feasible and accurate in estimating short-duration extreme design rainfalls for a given location of interest. Note that, for accuracy, only the estimated quantiles within the twice sample lengths were used for comparisons (i.e. up to 50-year and 25-year return periods for the calibration and validation respectively). 


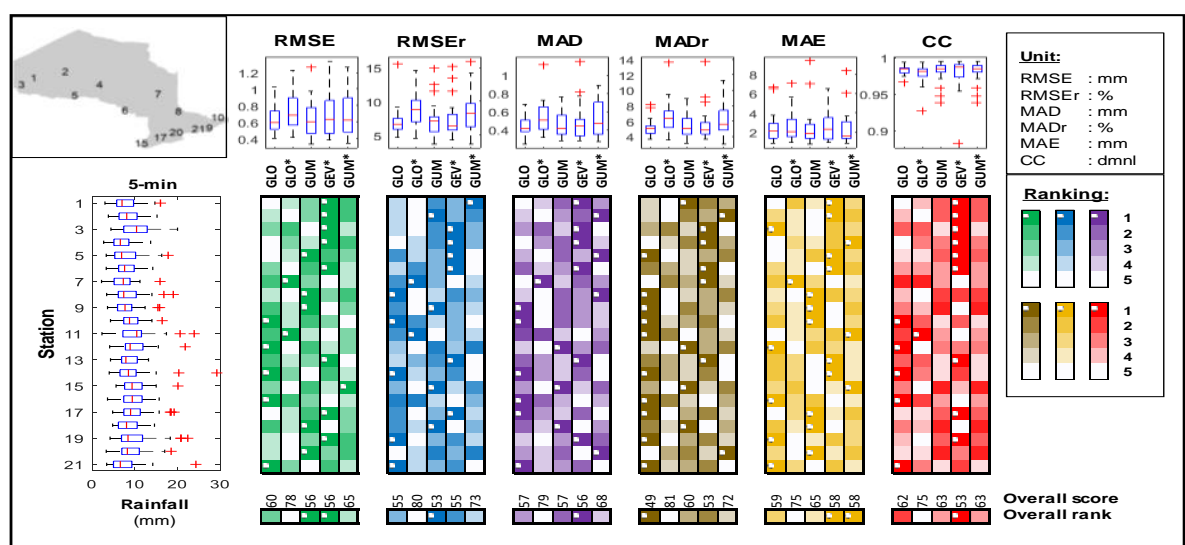

Figure 2: Comparison of GOF test results for the scaled and observed 5-min AMS based on six GOF indices. The test results are shown at the top in the form of boxplots. Ranking of the results (from 1 to 5) for each individual station are shown right below the boxplots. The observed 5-min AMS data is shown on the left.

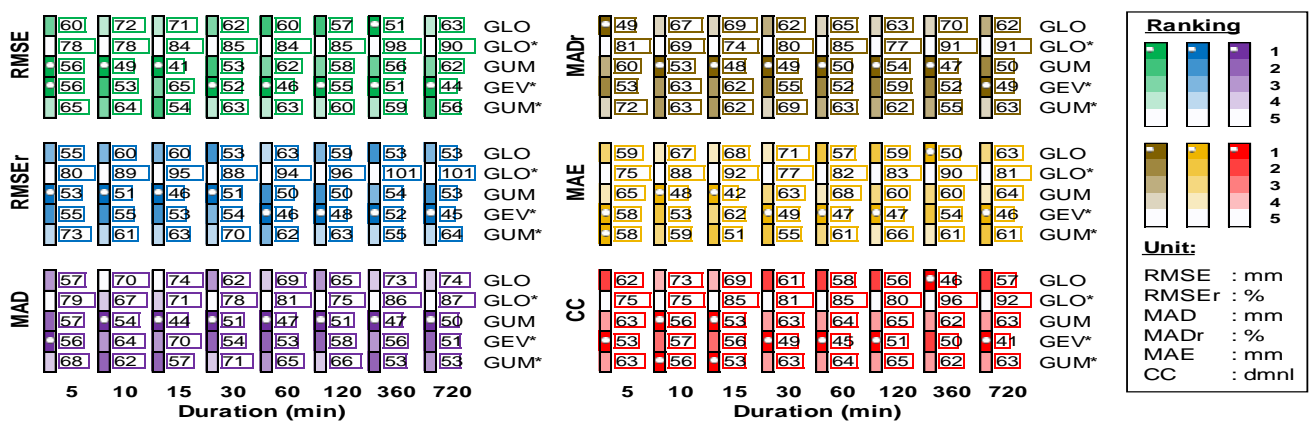

Figure 3: The overall rank of each model computed for all stations based on the six GOF criteria. The lowest scores (or the shortest bar) indicates the best models. GLO and GUM denote the scaling models based on PWMs, while GLO*, GEV*, and GUM* denotes the scaling models based on NCMs

(a)
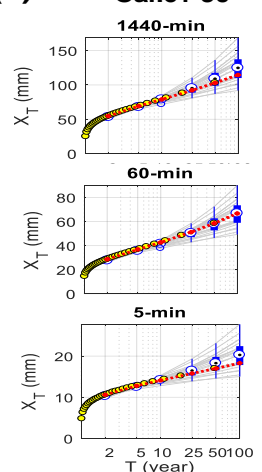
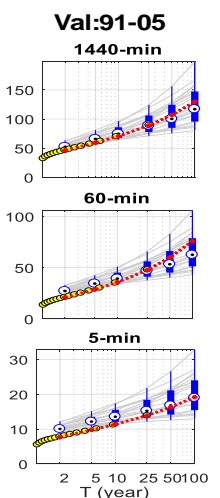

(b)
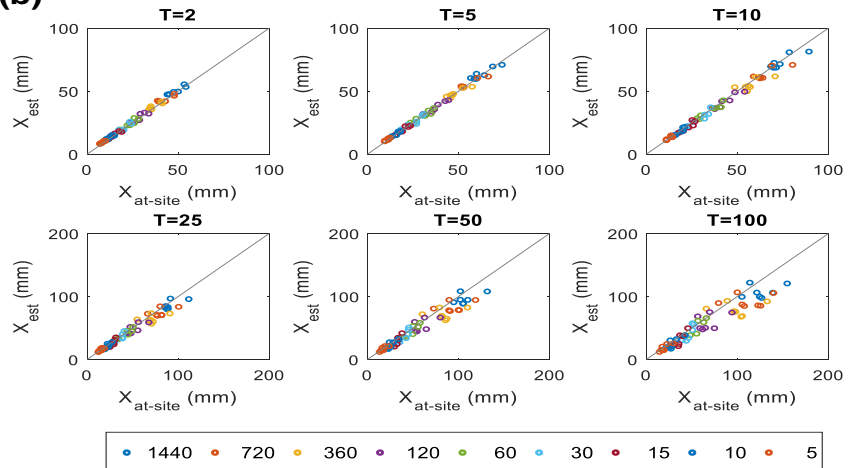

Figure 4: (a) Probability plots (PP) between the observed and estimated ERs at the Winsor station for the calibration (1961-1990) and validation (1991-2005) periods. Yellow circle markers show the empirical PPs while red cross markers and dash lines show the at-site frequency analysis plots. The gray lines and boxplots show the derived distributions of short-duration ERs and uncertainty from 21 climate simulation output based on the proposed scaling GLO/PWM model; (b) Q-Q plots of the estimated (Xest, $\mathrm{mm}$ ) and the at-site frequency analysis ( $\mathrm{X}_{\mathrm{at}-\mathrm{site}}, \mathrm{mm}$ ) ER quantiles using the GLO distribution for different rainfall durations (from $\mathrm{D}=5$ to 1440 minutes) and return periods ( $\mathrm{T}=2$ to 100 years) for the 1961-1990 calibration period of all seven study stations. 


\section{Conclusions}

Global and regional climate models along with different (spatial) statistical downscaling approaches have been extensively used to link projected climate change simulations to the daily extreme rainfalls at a given location of interest. Projected data of high temporal resolutions (i.e. subdaily or sub-hourly) at these local sites are often limited or unavailable due to current limitations on detailed physical modelling and computational capability of these climate models. A few statistical models based on the scale-invariance concepts have been introduced to overcome this problem. Nevertheless, there is still no general agreement as to which statistical model should be used for describing the distribution of annual rainfall extremes over a wide range of temporal scales (e.g., from one day to several minutes). The present study proposed therefore a novel scale-invariance GLO model for representing the AMS over different time scales.

The feasibility and accuracy of the proposed scaling GLO distribution using two different estimation methods GLO/NCM and GLO/PWM were assessed and compared with other existing models such as scaling GEV/NCM, GUM/PWM, and GUM/NCM. Results of this assessment using IDF data for nine different durations ranging (from 5-minutes to one day) from a network of 21 raingages located in Ontario (Canada) have indicated the comparable performance of the proposed scaling GLO/PWM as compared with other popular models such as the GEV/NCM. Hence, the GLO/PWM could be used to increase the confidence and reliability in the estimation of extreme design rainfalls in practice, especially in the UK where the GLO distribution has been recommended for modeling of extreme hydrologic variables.

Finally, the proposed scaling GLO model has been used for assessing the climate change impacts on the IDF relations using the observed ER data at seven raingages in Ontario and the downscaled regional ER (available at $25-\mathrm{km}$ regional scale) provided by the NASA. Results of this illustrative application have indicated the accuracy and robustness of the proposed scaling GLO/PWM model.

\begin{tabular}{lrrrrrrrrrrr}
\hline & \multicolumn{4}{c}{ Calibration period 1961-1990 } & & \multicolumn{4}{c}{ Validation period 1991-2005 } \\
\cline { 2 - 5 } \cline { 9 - 11 } T (year) & $\mathbf{2}$ & $\mathbf{5}$ & $\mathbf{1 0}$ & $\mathbf{2 5}$ & $\mathbf{5 0}$ & & $\mathbf{2}$ & $\mathbf{5}$ & $\mathbf{1 0}$ & $\mathbf{2 5}$ \\
\hline RMSEr (\%) & 4.9 & 4.8 & 7.2 & 13.0 & 17.7 & & 21.3 & 18.6 & 15.5 & 15.2 \\
MADr (\%) & 4.3 & 3.7 & 6.0 & 11.4 & 15.5 & & 16.4 & 15.4 & 12.9 & 12.1 \\
CC (dmnl) & 0.995 & 0.996 & 0.993 & 0.980 & 0.965 & & 0.979 & 0.980 & 0.975 & 0.956 \\
\hline
\end{tabular}

Table 1: Goodness-of-fit test results for both calibration and validation periods

\section{References}

[1] P. Willems, J. Olsson, K. Arnbjerg-Nielsen, S. Beecham, A. Pathirana, I.B. et al., Impacts of climate change on rainfall extremes and urban drainage systems, IWA Publishing, London, UK, 2012.

[2] V.V. Kharin, F.W. Zwiers, X. Zhang, M. Wehner, Changes in temperature and precipitation extremes in the CMIP5 ensemble, Clim. Change, 119 (2013) 345-357.

[3] S.P. Simonovic, A. Schardong, D. Sandink, R. Srivastav, A web-based tool for the development of Intensity Duration Frequency curves under changing climate, Environ. Model. Softw., 81 (2016) 136-153.

[4] X. Zhang, F.W. Zwiers, G. Li, H. Wan, A.J. Cannon, Complexity in estimating past and future extreme shortduration rainfall, Nature Geosci, advance online publication (2017).

[5] H.J. Fowler, S. Blenkinsop, C. Tebaldi, Linking climate change modelling to impacts studies: recent advances in downscaling techniques for hydrological modelling, Int. J. Climatol., 27 (2007) 1547-1578.

[6] D. Maraun, F. Wetterhall, et al., Precipitation downscaling under climate change: Recent developments to bridge the gap between dynamical models and the end user, Rev. Geophys., 48 (2010).

[7] E. Gooré Bi, P. Gachon, M. Vrac, F. Monette, Which downscaled rainfall data for climate change impact studies in urban areas? Review of current approaches and trends, Theor. Appl. Climatol., 127 (2017) 685-699. 
[8] V.T.V. Nguyen, T.D. Nguyen, A. Cung, A statistical approach to downscaling of sub-daily extreme rainfall processes for climate-related impact studies in urban areas, Wat. Sci. Tech.: Water Supply, 2007, pp. 183-192.

[9] S.M. Herath, P.R. Sarukkalige, V.T.V. Nguyen, A spatial temporal downscaling approach to development of IDF relations for Perth airport region in the context of climate change, Hydrol. Sci. J., 61 (2016) 2061-2070.

[10] J. Li, F. Johnson, J. Evans, A. Sharma, A comparison of methods to estimate future sub-daily design rainfall, Adv. Water Resour., 110 (2017) 215-227.

[11] T. Lee, T. Park, Nonparametric temporal downscaling with event-based population generating algorithm for RCM daily to hourly: Model development and performance evaluation, J. Hydrol., 547 (2017) 498-516.

[12] R.L. Wilby, C.W. Dawson, The Statistical DownScaling Model: insights from one decade of application, Int. J. Climatol., 33 (2013) 1707-1719.

[13] M. Khalili, V.T. Van Nguyen, An efficient statistical approach to multi-site downscaling of daily precipitation series in the context of climate change, Climate Dynamics, (2016).

[14] A.T. Werner, A.J. Cannon, Hydrologic extremes -- an intercomparison of multiple gridded statistical downscaling methods, Hydrol. Earth Syst. Sci., 20 (2016) 1483-1508.

[15] R.L. Wilby, C.W. Dawson, E.M. Barrow, SDSM - A decision support tool for the assessment of regional climate change impacts, Environ. Model. Softw., 17 (2002) 147-159.

[16] P. Hubert, Multifractals as a tool to overcome scale problems in hydrology, Hydrol. Sci. J., 46 (2001).

[17] D. Veneziano, P. Furcolo, Multifractality of rainfall and scaling of intensity-duration-frequency curves, Water Resour. Res., 38 (2002) 42-41-42-12.

[18] D. Veneziano, C. Lepore, The scaling of temporal rainfall, Water Resour. Res., 48 (2012) n/a-n/a.

[19] S. Lovejoy, D. Schertzer, The Weather and Climate: Emergent Laws and Multifractal Cascades, Cambridge University Press, Cambridge, 2012.

[20] V.K. Gupta, E. Waymire, Multiscaling properties of spatial rainfall and river flow distributions, Journal of Geophysical Research: Atmospheres, 95 (1990) 1999-2009.

[21] V.T.V. Nguyen, T.D. Nguyen, H. Wang, Regional estimation of short duration rainfall extremes, Water Science and Technology, 37 (1998) 15-19.

[22] V.T. Nguyen, T.D. Nguyen, F. Ashkar, Regional frequency analysis of extreme rainfalls, Water science and technology, 45 (2002) 75-81.

[23] M. Menabde, et al., A simple scaling model for extreme rainfall, Water Resour. Res., 35 (1999) 335-339.

[24] P.-S. Yu, T.-C. Yang, C.-S. Lin, Regional rainfall intensity formulas based on scaling property of rainfall, J. Hydrol., 295 (2004) 108-123.

[25] J. Bougadis, K. Adamowski, Scaling model of a rainfall intensity-duration-frequency relationship, Hydrol. Processes, 20 (2006) 3747-3757.

[26] J. Blanchet, D. Ceresetti, G. Molinié, J.D. Creutin, A regional GEV scale-invariant framework for IntensityDuration-Frequency analysis, J. Hydrol., 540 (2016) 82-95.

[27] A.K. Bairwa, R. Khosa, R. Maheswaran, Developing intensity duration frequency curves based on scaling theory using linear probability weighted moments: A case study from India, J. Hydrol., 542 (2016) 850-859.

[28] H. Ghanmi, Z. Bargaoui, C. Mallet, Estimation of IDF relationships according to the property of scale invariance and regionalization analysis in a Mediterranean coastal area, J. Hydrol., 541 (2016) 38-49.

[29] M.T. Vu, V.S. Raghavan, S.Y. Liong, Deriving short-duration rainfall IDF curves from a regional climate model, Natural Hazards, (2016).

[30] J.R.M. Hosking, J.R. Wallis, Regional Frequency Analysis: An Approach Based on L-Moments, Cambridge University Press, Cambridge, UK, 1997.

[31] WMO, Guide to hydrological practices, volume II: Management of water resources and application of hydrological practices, 6th edition, World Meteorological Organization, Geneva, Switzerland, 2009.

[32] T.-H. Nguyen, S. El Outayek, et al, A systematic approach to selecting the best probability models for annual maximum rainfalls - A case study using data in Ontario (Canada), J. Hydrol., 553 (2017) 49-58.

[33] J.L. Salinas, A. Castellarin, et al., Regional parent flood frequency distributions in Europe - Part 1: Is the GEV model suitable as a pan-European parent? Hydrology and Earth System Sciences, 18 (2014) 4381-4389.

[34] B. Thrasher, E.P. Maurer, C. McKellar, P.B. Duffy, Technical Note: Bias correcting climate model simulated daily temperature extremes with quantile mapping, Hydrol. Earth Syst. Sci., 16 (2012) 3309-3314.

[35] T.-H. Nguyen, V.-T.-V. Nguyen, Assessment of climate change impacts on extreme rainfall IDF relations using downscaled CMIP5 climate projections, EWRI 2018, Minnesota, US, 2018. 